\title{
A Proof-of-Concept, Rover-Based System for Autonomously Locating Methane Gas Sources on Mars
}

\author{
Laszlo-Peter Berczi*, Jonathan D. Gammell*, Chi Hay Tong*, Michael Daly ${ }^{\dagger}$, and Timothy D. Barfoot* \\ *Institute for Aerospace Studies \\ University of Toronto, Toronto, Canada \\ \{peter.berczi, jon.gammell, chihay.tong, tim.barfoot $\} @$ utoronto.ca \\ ${ }^{\dagger}$ Dept. of Earth and Space Science and Engineering \\ York University, Toronto, Canada \\ dalym@yorku.ca
}

\begin{abstract}
This paper presents a proof-of-concept, roverbased system to locate the source(s) of methane gas on Mars. A distributed open-path spectrometer is mounted on a rover and pointed at several retroreflective signs to measure the lineof-sight methane concentration between the rover and sign. By moving the rover around and accumulating such measurements, the location of a methane source can be determined. The system was integrated and tested over a 2 kilometre traverse in an analog mission at the Canadian Space Agency on the Mars Emulation Terrain. Engineering results suggest that total driving distance is comparable to other similar methods, but by using a network of reusable paths, the total distance driven in new terrain can be reduced by a factor of five. Autonomous pointing of signs was successful $80 \%$ of the time. Future work includes combining the engineering and science data from the analog mission to better understand how to locate methane sources.
\end{abstract}

Keywords-autonomous; rover; spectrometer; sign; methane

\section{INTRODUCTION}

On Earth, 90\% of atmospheric methane is produced by living organisms (biogenic), with only $10 \%$ coming from non-living processes (abiogenic) [1]. As a result, scientists hope that Martian atmospheric methane, recently discovered by the Planetary Fourier Spectrometer on the Mars Express Orbiter [2], by several Earth-based telescopes [1], and by the Fourier Transform Spectrometer at the Canada-FranceHawaii Telescope [3] can provide clues about the existence, past or present, of life on Mars.

Methane gas has been found to occur in extended plumes, released in distinct regions on the surface of the red planet [1]. The origin of the gas is unknown, but many aboveground processes have been eliminated (e.g., processes in the atmosphere, volcanic emissions, and cometary impacts). Scientists therefore hypothesize that the gas is seeping into the atmosphere from underneath the surface [3].

If these seeps could be located and approached at close range, scientists could then perform experiments to determine whether or not the methane gas on Mars is biogenic. Previous Martian surface exploration missions, such as the Mars Exploration Rovers (MERs) and the Mars Science

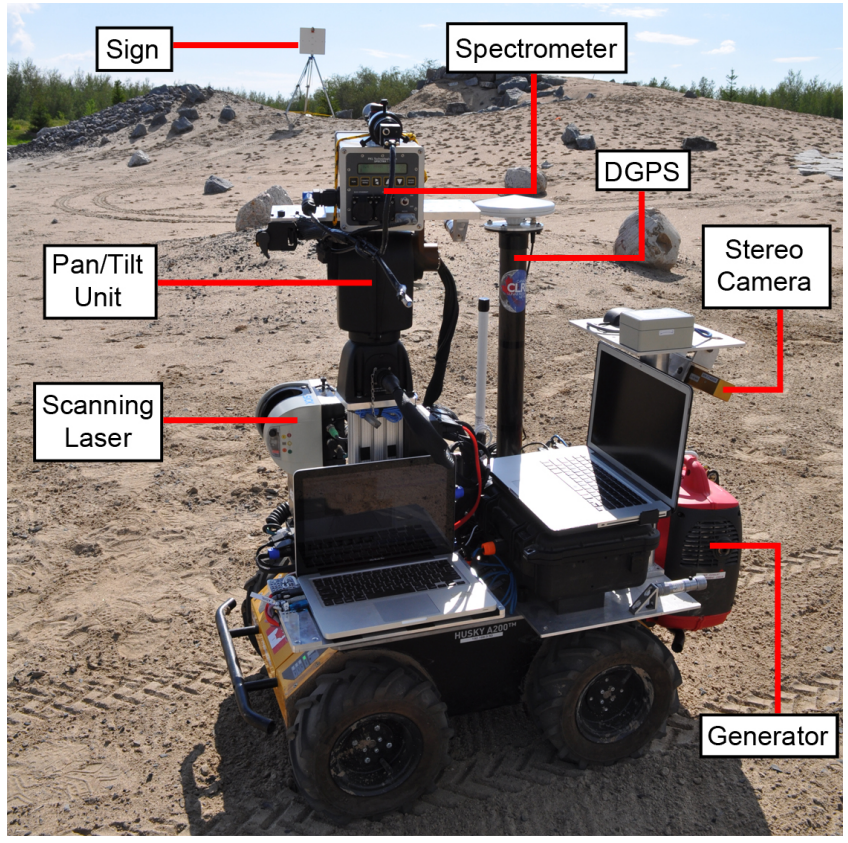

Figure 1. Clearpath Husky A200 rover with science and engineering payloads mounted. System shown pointing spectrometer at a retroreflective sign to measure the gas concentration along the line-of-sight between the spectrometer and sign. Differential GPS (DGPS) is used as groundtruth for the position of the rover. A 1 kilowatt generator is mounted to enable long-term operation of the rover.

Laboratory (MSL) have typically relied on rovers for their mobility, ability to carry payloads, and durability (MER Opportunity continues to operate almost 9 years after its planned lifespan of $90 \mathrm{sol}$ ). Such a rover (with appropriate payloads) would be well suited to explore the surface of Mars in search of a source of methane gas, and then to perform in-situ experiments to determine the origin of the gas.

Rovers equipped with close-range sensors (such as ground-penetrating RADAR [4] or a fluorescence imager [5]) could be driven over an area to locate a methane seep. 


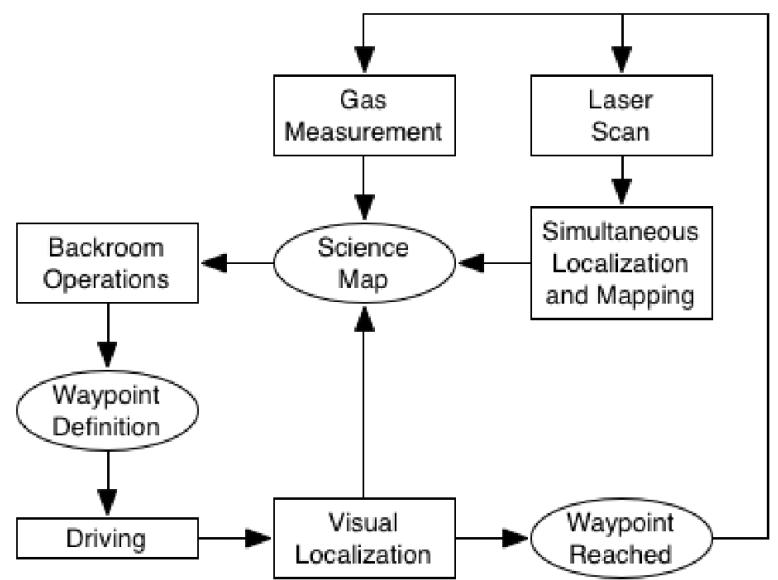

Figure 2. The general system architecture. The system operates as a cycle, beginning with the backroom operations team, until the scientists can estimate the location of the methane source. Rectangles represent processes and ellipses represent data.

Tunstel et al. [6] argue that using a distributed open-path spectrometer with a distant retroreflective sign improves upon methods that use close-range sensors, resulting in better coverage with less distance driven. Anderson et al. [7] implement that distributed system on a rover and present the results from small-scale tests showing that the system can successfully measure gas concentrations along the lineof-sight between the rover and a sign. Tolson et al. [8] also show results for using an open-path spectrometer with a distant retroreflective sign in outdoor conditions to successfully detect the line-of-sight gas concentration.

Anderson et al. [9] then tailor the single-rover system to Mars exploration, whereby a Mars lander is used as a $360^{\circ}$ retroreflector central to the target survey area. A spectrometer mounted on a pan/tilt unit is automatically aimed at the retroreflector using a vision algorithm to track its location. They present circular and spiral driving patterns and an algorithm for determining the location of the gas source from periodic measurements. Tunstel et al. [10] employ the same method and show computer simulations of the driving patterns and gas localization algorithm. Later, they go on to fully integrate the above systems on a real rover. Indoor lab experiments and outdoor sandbox experiments are conducted validating the design. The experiments were limited to circular areas six metres in diameter [11].

These lander-centered systems are limited to surveying the area in the vicinity of the lander. If the lander is not near the methane source, then the circular pattern that the rover needs to drive may be prohibitively large to complete. Furthermore, obstacles in the terrain may make circular or spiral driving patterns impossible to drive.

Our approach is similar to that of Tunstel et al., in that we use a single rover-based system employing an open- path spectrometer, but instead of one retroreflective sign, we employ multiple signs. This allows for more arbitrary paths to be driven in the test area, avoiding obstacles if necessary. Our driving distance is expected to be reduced using multiple signs because multiple measurements in different directions can be gathered at each location, allowing for more strategic route planning. The design and implementation of an engineering system to support a Martian methane gas detection mission is presented in this paper.

Section II presents the engineering requirements for the system, derived from the scientific motivation of detecting methane gas on Mars. The design of a rover-based system that meets these requirements is presented in Section III. A description of and the results from an analog mission to test the system are presented in Section IV and Section V. Section VI presents a discussion of the results and outlines future work related to this paper.

\section{REQUIREMENTS}

In order to successfully determine the location of a gas source on the surface of Mars as outlined in Section I, certain science and engineering tasks need to be completed. Science tasks include analyzing gas measurements, deciding where to take new measurements, and eventually combining the measurements to estimate the location of the source. The engineering task is to gather the required measurements for the scientists. This engineering task can be defined in terms of the following requirements, many of which need to be autonomous due to communication constraints present when operating on Mars:

1) The system will carry science and engineering payloads, including an open-path spectrometer.

2) The system will receive waypoints from a remote backroom.

3) The system will autonomously move between waypoints.

4) The system will autonomously point the spectrometer at each retroreflective sign.

5) The system will autonomously measure line-of-sight gas concentrations between the rover and a retroreflective sign.

6) The system will create a global map of the environment augmented with line-of-sight gas measurements.

These high-level requirements fully define the system outlined in this paper. The design decisions made in order to meet these requirements are outlined in Section III.

\section{Methodology}

The system architecture can be seen in Figure 2. A rover is chosen that is large enough to fit all of the required payloads and powerful enough to drive fully loaded (Requirement 1). It communicates with a remote backroom to receive waypoints and instructions to perform at those waypoints (Requirement 2). To get to the desired locations 


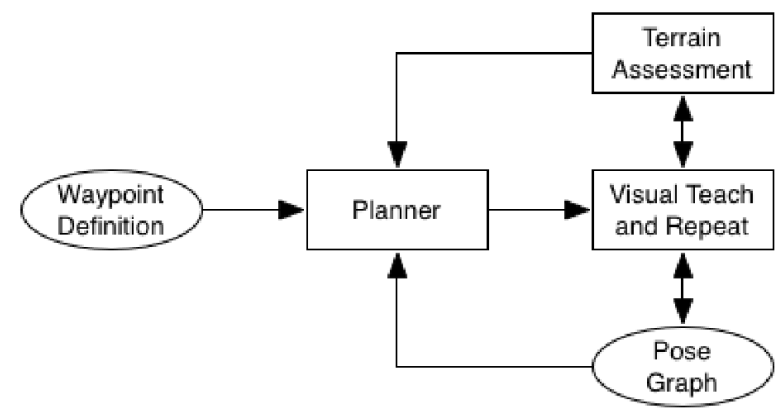

Figure 3. The Visual Teach and Repeat (VT\&R)-based Network of Reusable Paths (NRP) pipeline used to navigate to user-specified points of interest while maintaining the ability to return to any previously visited location. Given the existing network, a path is planned to the goal and driven as a teach-pass by VT\&R. During the teach pass, the terrain is continually assessed for obstacles and general feasibility and if at any point the path is found to be hazardous, the path is stopped and a new solution is planned. This process is continued until the rover arrives at the goal. Rectangles represent processes and ellipses represent data.

accurately, the system localizes against a visual map that it builds up as it drives (Requirement 3). Once it arrives at a waypoint, the system may deploy a sign. The system then takes a laser scan of the environment, which is used to determine the locations of previously deployed signs and build a geometric map of the environment (Requirement 6). A spectrometer, which is mounted on a pan/tilt unit (PTU) with a pan range of $360^{\circ}$ and a tilt range of $-30^{\circ}$ to $45^{\circ}$, then points at the signs (Requirement 4 ) and measures the gas concentration along the line of sight between itself and the signs (Requirement 5).

\section{A. Rover Navigation Using a Network of Reusable Paths}

Accurately traversing to specified waypoints (Requirement 3 ) is a challenging problem in robotics, especially in GPS-denied environments such as extra-terrestrial planets. It has been demonstrated that a class of algorithms known as visual teach and repeat (VT\&R) can be used to autonomously repeat previously driven paths with accuracy in the tens of centimeters [12]. These algorithms store rover traverses as a network of relative odometric measurements generated from stereo visual odometry (VO) and tagged with unique visual features. After the path has been driven once, the rover is capable of repeating it using nothing more than a stereo camera. The algorithm matches the visual features observed in the current scene with those recorded during the teach pass, and using their 3D offset, calculates the necessary control input to steer the rover along the previously driven path. The accuracy is sufficient that, for large sections of the traverse, the rover will drive exactly in its previous tracks.

Network of Reusable Path (NRP) systems provide a way to leverage the strengths of VT\&R to create a system for long-term autonomous navigation [13]. They do so by replacing the manual teach phase in VT\&R with an autonomous exploration algorithm that makes use of any preexisting network to plan a path to a user-specified goal. Given the goal as a relative transformation from a previous rover pose, NRP plans a path from the current rover location to the specified location using the pre-existing network and any terrain information available (Figure 3 ). The resulting path can be any combination of on-network or off-network driving. The on-network path is calculated using graphsearches on the network and is a VT\&R repeat traverse, while the off-network path is generated using a rapidlyexploring random tree (RRT) planner [14] and is a VT\&R teach traverse.

As off-network drives are VT\&R teach passes, the newly driven routes are available as additional existing paths in any new planning problems. During these exploration phases, the rover is using $\mathrm{VO}$ to drive an open-loop distance to the goal; however, during repeat phases, the localization error is rolled back as the robot recognizes where it has previously driven. As a result, the total localization error in a network is a function of the network length and not the distance driven by the robot. Also, the rover can return to any point on the network repeatedly, meaning the scientists can return to and increase the number of measurements at any point that the rover has already visited with low risk. NRP systems have been successfully used in lunar analog sample-return missions [15].

\section{B. Science and Engineering Data Collection}

Data collection occurs in two distinct stages after reaching a waypoint. First, a laser scan is obtained using a panning lidar. In consideration of the slow acquisition rate of the lidar, the $360^{\circ} \times 190^{\circ}$ scans are obtained with the rover stationary. This approach ensures local consistency of the point cloud by avoiding the complexity of motion distortion within a single scan.

The locations of the retroreflective signs are determined autonomously by analyzing the intensity image from the laser scan (Figure 4). The signs are very reflective, and thus produce high-intensity spots that are easy to identify in the image. In cases where a sign is occluded from the laser scan by the rover, the location of the sign is estimated by using its last known location and an estimate of the rover's motion since the last observation.

Once an estimate for each sign location has been obtained, the system autonomously points the open-path spectrometer at the sign (Requirement 4). Feedback of the signal strength from the spectrometer is used to determine whether or not the sign is being sighted (a miss can occur due to a misalignment of the lidar and spectrometer frames or due a poor estimate of the sign location). If the spectrometer misses the sign, a spiral search algorithm is used to search the area near the estimated location of the sign. If the search algorithm fails, the remote backroom has the option to sight the spectrometer remotely using a camera mounted on a 


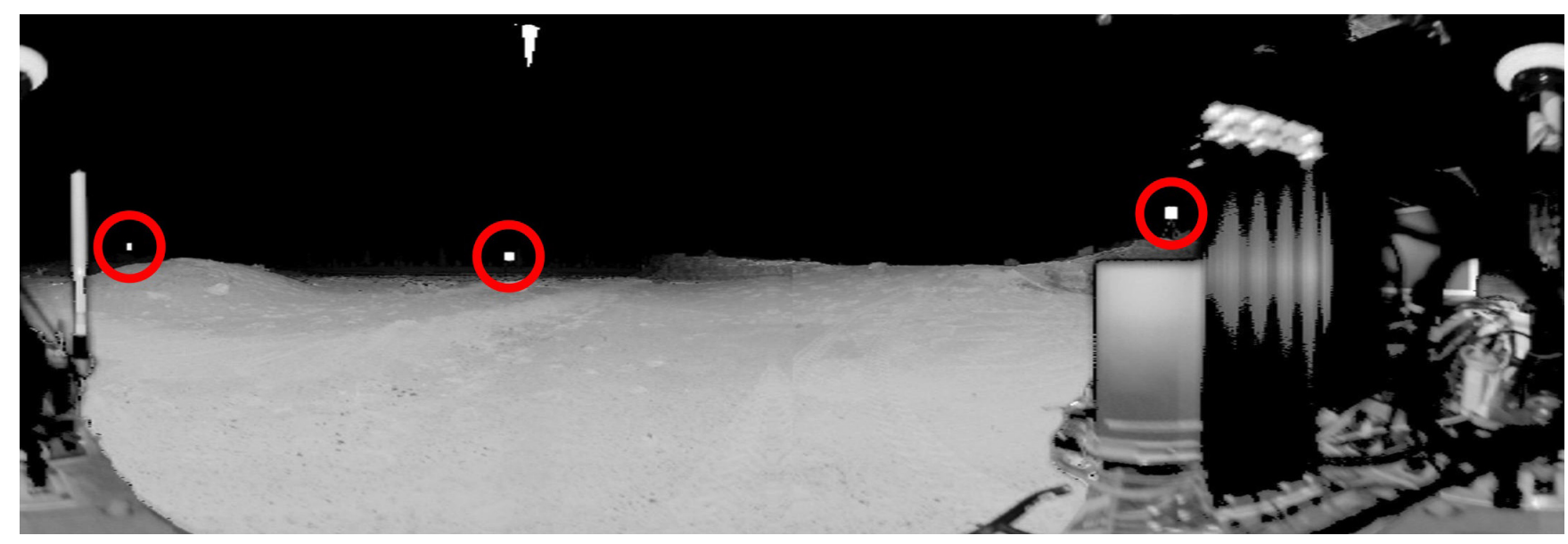

Figure 4. A sample cropped laser intensity image with retroreflective signs highlighted by red circles. Due to the retroreflective coating on the signs, they appear as very bright spots despite their long range and are easy to identify. The occlusions on the right and left sides of the image are due to the rover. The white streak in the sky is caused by the Sun. The cause of this artifact and its uses are discussed in [16].

scope. Once the signal strength returned by the spectrometer is high enough, line-of-sight gas concentration readings are taken (Requirement 5).

\section{SLAM}

In order to combine the lidar scans and generate a global 3D map of the environment (Requirement 6), we need to estimate the six-degree-of-freedom (6DOF) rover pose at each scan location. By adding the locations of the signs to the state we wish to estimate, we can formulate the task as a batch simultaneous localization and mapping (SLAM) problem. We employ a sparse-feature-based approach, where distinct geometric features are identified in each scan, and utilize visual odometry data in case of feature scarcity. This SLAM architecture is illustrated in Figure 5. While the complete details of the SLAM framework can be found in [17], we provide a brief overview in this section.

Given a collection of $3 \mathrm{D}$ point clouds, we begin by identifying distinct geometric features in each scan. In our

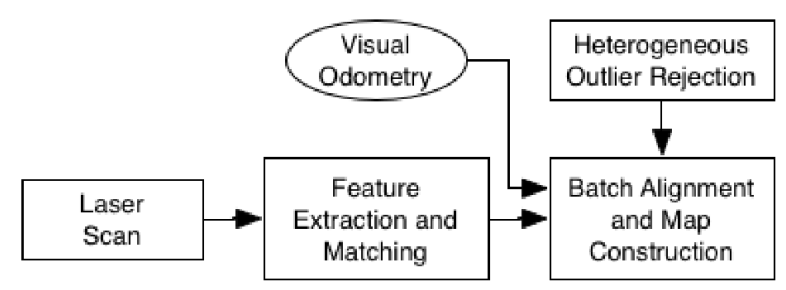

Figure 5. The laser-based 3D SLAM architecture used to estimate the rover poses and sign locations. Retroreflective sign positions and distinct geometric features are extracted from each laser scan, and are utilized alongside visual odometry measurements in a batch alignment algorithm that incorporates heterogeneous outlier rejection for robustness. After convergence, the scans are overlaid according to the pose estimates to construct a map. Rectangles represent processes and ellipses represent data. implementation, peaks in the terrain are chosen to serve as the distinctive interest points due to their ease of extraction and visibility at long ranges. An example of the extracted features is provided in Figure 6.

Matching these peak features between scans is a multistep process. For each pair of scans, feature constellations are matched using the Data-Aligned Rigidity-Constrained Exhaustive Search (DARCES) [18] algorithm, which generates a set of alignment hypotheses. This constellationmatching process can be viewed as seeking sets of similar triangles between scans; however, due to the large uncertainties in the feature extraction process, numerous incorrect hypotheses are produced by the DARCES algorithm. For validation, we overlay the dense data according to each alignment hypothesis, and compute a quality metric based on the average mismatch in the overlapping regions. A threshold is then applied on the scores, resulting in a set of valid hypotheses. Finally, the data association process is concluded by resolving the valid constellation matches into individual feature associations.

In addition to the peak features, we identify the retroreflective signs in each scan for use in sign pointing, as well as in the SLAM formulation. We perform simple clustering to identify the signs, and utilize prior estimates for data association. The use of the retroreflective signs in the SLAM formulation benefits estimator accuracy, and meets the sign localization requirement for spectrometer pointing.

In addition to the point features obtained in each scan, we utilize the VO estimates of motion between scan stops. This provides flexibility in the rover traverse in case of feature scarcity or lack of scan overlap. Since these estimates are already computed for navigation, it is straightforward to store them for later use.

Finally, we align the scans by utilizing a batch alignment formulation. By considering the SLAM task as an opti- 


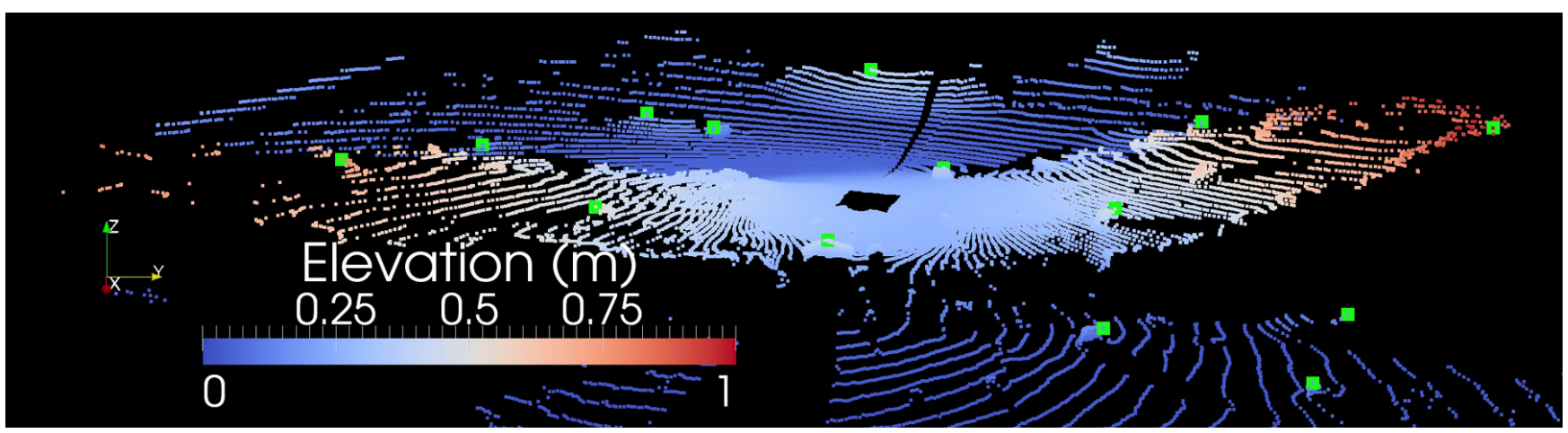

Figure 6. A sample point cloud obtained by the panning laser rangefinder. The scan was taken from the center of the image, with the rectangular cut-out corresponding to the rover's footprint, and the green squares indicating the detected peak features.

mization problem, we seek a globally consistent estimate of all rover poses and feature positions that best match the measurement data available. For robustness, we augment the alignment algorithm with heterogeneous outlier rejection to address the possibility of outliers in either measurement type [19], resulting in estimates for the rover poses, geometric feature positions, and retroreflective sign locations.

The rover tracks, as estimated by VO, will not be consistent with the rover poses at the scan locations, as estimated by the SLAM solution. To provide the scientists with a consistent estimate for the rover's position, the VO estimates are relaxed with the SLAM estimates as constraints in the publicly available GTSAM library ${ }^{1}$ from the Georgia Institute for Technology. The VO estimates are relative odometric measurements representing the rover tracks, and the SLAM estimates are absolute pose measurements at the scan locations. GTSAM provides adjusted odometric measurements such that the rover tracks are consistent with the SLAM estimates.

These improved VO estimates are only used to present a coherent picture to backroom operators through the groundstation, and are not required by the rover for localization. This provides the operators with a better understanding of the rover's path through the 3D point clouds collected by the lidar.

\section{Remote Backroom Software}

At each waypoint, the aligned laser scans are sent to the remote backroom along with the line-of-sight gas concentration measurements. There, the data are processed by a science team using custom backroom software. They visualize the outputs of the SLAM algorithm by plotting the scan poses, rover tracks, sign locations, and point clouds according to the alignment estimates. The interface, depicted in Figure 7, permits strategic decisions based on the geometry of the terrain in combination with other tools to analyze the spectrometer data.

\footnotetext{
${ }^{1}$ https://collab.cc.gatech.edu/borg/gtsam/
}

In addition to visualizing previously obtained data, the backroom software also serves as the planning interface for specifying future rover waypoints. Scientists provide a new waypoint as an input to the system and the process repeats until the source of the methane is located.

\section{AnAlOG Mission}

An analog mission to validate the methodology described in Section III was conducted at the Canadian Space Agency (CSA) on the Mars Emulation Terrain (MET), located in Montréal, Canada. The mission consisted of two separate scenarios in which the goal was to determine the location of an artificial gas source placed on the terrain. The location of the gas source was not disclosed to the remote backroom, who could only communicate to the field via the backroom software (Figure 7). Signs were pre-deployed by humans to speed up the testing process.

\section{A. Mars Emulation Terrain Specifications}

The MET is an artificially created environment that emulates the terrain a rover might encounter on Mars (Figure 9).

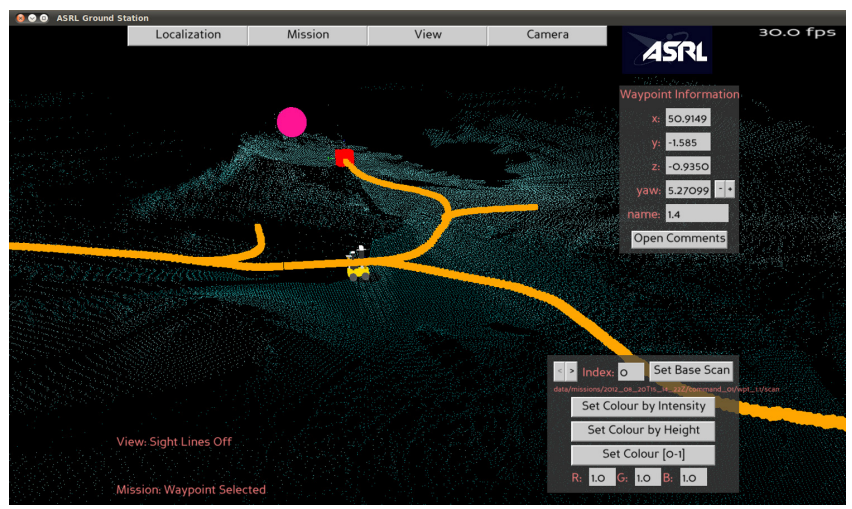

Figure 7. A screenshot of the backroom software depicting a model of the rover, point cloud map, rover tracks in orange, and a magenta sphere marking a retroreflective sign position. This software is used by the science team for visualizing the exploration data and manual waypoint planning. 


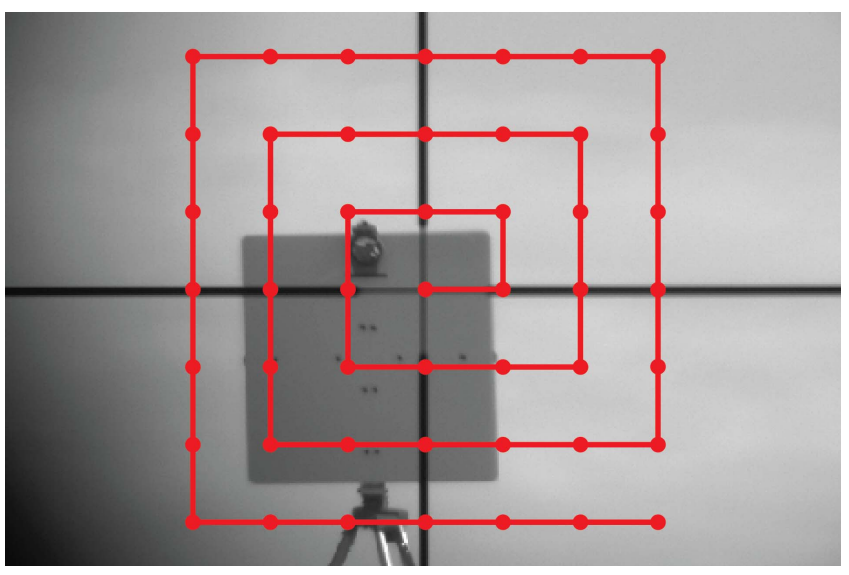

Figure 8. Retroreflective sign targeted by spectrometer. Signs are mounted on a tripod. Retroreflective cube mounted at top of sign. In case of a miss, search pattern (shown in red) is used to search for sign.

The surface composition is sandy, with rocks of various sizes littering the area. The dimensions of the MET are $120 \times 60$ metres, and there are several elevation changes throughout the area.

Artificial gas sources were placed on the terrain for the analog mission. The sources were covered with rocks and connected to methane gas bottles that were hidden outside of the terrain so that their locations would not be discernible using lidar scans.

\section{B. Rover Platform}

The Husky A200 was the chosen rover platform, the same rover platform as in [20]. It is a 4-wheel differential drive robot equipped with two motors. The motors are electric and the rover is outfitted with a 1000 watt gas generator for long-term operation.

The rover is equipped with several payloads required for the mission. A differential GPS receiver (DGPS) is used to collect groundtruth position data at $1 \mathrm{~Hz}$. A real time kinematic (RTK) base station was deployed as well to correct the GPS readings when possible. The stereo VO algorithm used a Point Grey Bumblebee XB3 stereo camera mounted on the rover. The camera is capable of $1280 \times 960$ resolution at $15 \mathrm{~Hz}$. During the experiment, frames were captured at $10 \mathrm{~Hz}$ due to the time required to process each image. A SICK scanning laser rangefinder is mounted on a Schunk pan/tilt unit to enable it to take full spherical range scans. Finally, a PKL Technologies Spectra-1 laser spectrometer is mounted on a Directed Perception pan/tilt unit (PTU) with a resolution of 0.0075 degrees per step in order to point the spectrometer at retroreflective signs and obtain a gas concentration measurement.

\section{Signs and Spectrometer}

For this proof-of-concept mission, four $50 \times 50 \mathrm{~cm}$ signs were constructed and coated with $3 \mathrm{M}$ retroreflective

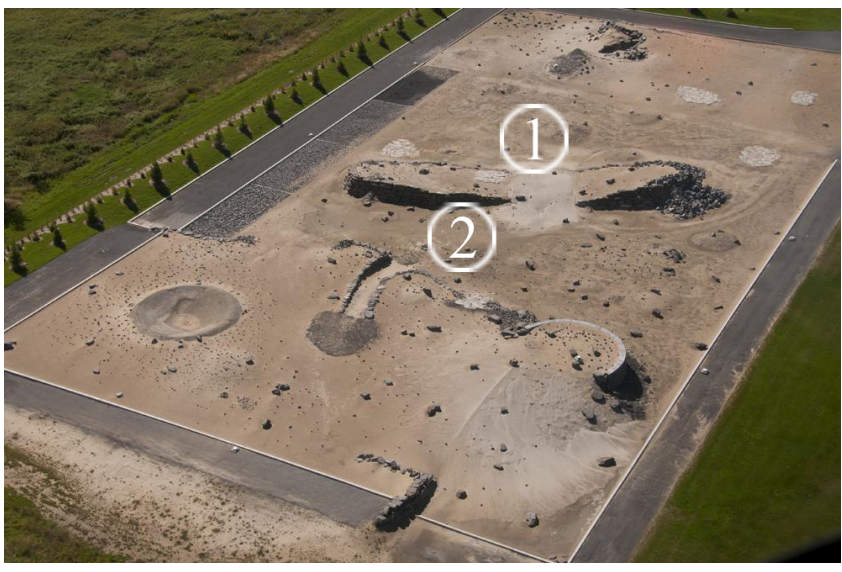

Figure 9. The Mars Emulation Terrain at the Canadian Space Agency. Artificial methane gas source locations for an analog mission are circled in white. The area is $120 \times 60$ metres in dimension.

sheeting. They were mounted on tripods to allow for panning and tilting so that they can be pointed directly at the spectrometer for maximum reflectivity. The reflectivity of the signs dropped off anisotropically as the incidence angle of the laser increased. The signs were therefore mounted to allow for greater angles of incidence in the vertical direction, since aligning the signs in the pan axis was easier than in the tilt axis.

The Spectra-1 spectrometer measures line-of-sight gas concentrations as well as signal strength and can operate at ranges of over 1 kilometre in ideal conditions. However, a minimum signal strength of $10 \%$ was required, and it was found that the maximum range at which this could be accomplished was 30 metres using the $3 \mathrm{M}$ retroreflective sheeting. Retroreflective cubes were therefore mounted on each sign in case the signal strength was too low using the sign alone. For a real mission, better quality, larger retroreflective cubes would be mounted in a circle allowing for full $360^{\circ}$ reflectivity with no panning of the signs.

\section{RESUlts}

The results from the analog mission in Section IV are presented in two main categories: (i) driving distance; and (ii) sign pointing.

Driving distance is comprised of two statistics. The NRP length is the distance traveled by the robot if repeat traverses along the NRP are ignored; it is the distance traveled by the rover into 'new' terrain. The total distance driven, on the other hand, is the sum total of all driving done by the rover, repeat traverses included (Table I). All distances were measured by the DGPS receiver on the rover and include the driving distance required to deploy the signs.

Sign pointing statistics were partitioned based on whether the sign was sighted using the initial estimate for its position, using the search pattern after an initial estimate, or using human intervention from the backroom. The statistics are 
Table I

DRIVING DISTANCE AND NETWORK LENGTH

\begin{tabular}{lccc}
\hline & Scenario 1 & Scenario 2 & Total \\
\hline NRP length $(\mathrm{m})$ & 148 & 235 & 383 \\
Total driving distance $(\mathrm{m})$ & 563 & 1170 & 1733 \\
\hline
\end{tabular}

further partitioned based on whether the initial estimate for the sign was determined using the lidar intensity image or not.

Table II

SUCCESSFUL SIGN POINTS FOR ANALOG MISSION

\begin{tabular}{lccc}
\hline & Scenario 1 & Scenario 2 & Total \\
\hline Initial Estimate & 5 & 7 & 12 \\
Search Pattern & 20 & 13 & 33 \\
Human & 7 & 4 & 11 \\
\hline Initial Estimate (intensity) & 2 & 4 & 6 \\
Search Pattern (intensity) & 12 & 5 & 17 \\
Human (intensity) & 1 & 1 & 2 \\
\hline Initial Estimate (no intensity) & 3 & 3 & 6 \\
Search Pattern (no intensity) & 5 & 8 & 13 \\
Human (no intensity ) & 6 & 3 & 9 \\
\hline
\end{tabular}

\section{Discussion AND FUtURE WORK}

Other methods described in Section I propose driving concentric circles in order to locate the methane source. Such a pattern is estimated to require a total driving distance of between 1000 metres (with 10 metres between circles) and 11000 metres (with 1 metre between circles). Furthermore, the topography of the terrain (Figure 10) shows that such patterns would be impossible to drive.

The total driving distance using our system was at the low end of the range for other methods (even lower for Scenario 1). In addition, most (more than 80\%) of the driving using our system consisted of repeat traverses, whereas other methods drive over new terrain the entire time. As a result, our system drives one fifth as much new terrain, which is considerably more expensive in terms of mission time than terrain that has already been driven on successfully.

Overall, the system was able to autonomously sight the signs with the spectrometer in $80 \%$ of the pointing attempts. The system was able to point at a sign directly (without a search algorithm) $21 \%$ of the time.

For the subset of attempted sign points where the sign was visible in the lidar intensity image, the success rate for autonomous pointing is considerably higher at $92 \%$. In contrast, the subset of attempted points where the sign could not be seen in the intensity image, a lower success rate for autonomous pointing of $68 \%$. In other words, the system performs better when the locations of the signs can be measured directly by the laser rather than estimated from prior knowledge and rover motion.

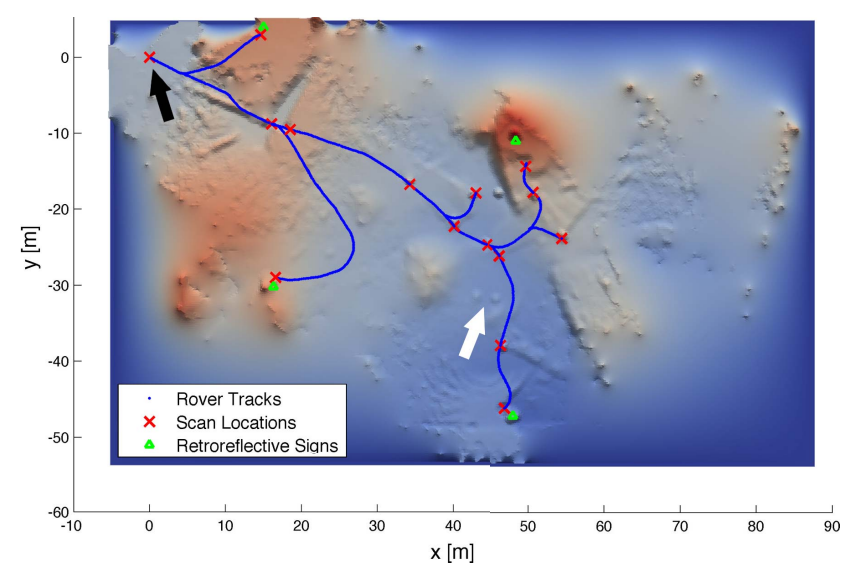

(a) Scenario 1

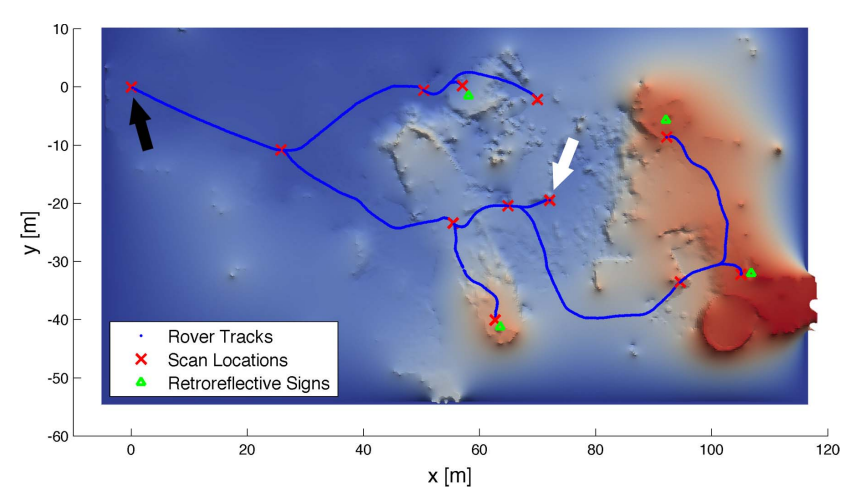

(b) Scenario 2

Figure 10. Rendered overhead view of the network of reusable paths and meshed pointclouds for: (a) Scenario 1; and (b) Scenario 2. Blue terrain indicates lower elevations, red terrain indicates higher elevations. Start location is indicated with a black arrow, commanded waypoints are indicated in red, sign locations are indicated in green, and the gas source is indicated with a white arrow.

The science team was able to use the engineering data to determine the location of the methane source successfully in Scenario 2, and drove the rover there (Figure 10). Wind and inexperience with the system were factors in the failure to locate methane in Scenario 1. Future work includes gaining a better understanding of how to determine the location of a gas source from the line-of-sight gas measurements. More knowledge regarding this process may allow the science team to command better waypoints to the rover, reducing the total rover driving distance and mission time.

\section{CONCLUSION}

A rover-based system was designed to meet a set of engineering requirements, outlined in Section II, that were derived from scientific goals presented in Section I. The system was successfully integrated and tested in an analog mission on Mars-like terrain.

The results suggest that our system requires less driving 
than other comparable systems, but whereas others are driving on new terrain the whole time, less than $20 \%$ of our driving is on new terrain. The system was able to autonomously sight retroreflective signs $80 \%$ of the time.

By combining the science and engineering data, an accurate global 3D map of the environment (both in terms of geometry and gas concentrations) was created. The map, along with accurately superimposed rover tracks, was successfully used in custom software by an operations backroom to give high-level directives to the system. A remote science team was able to successfully use the system to determine the location of a gas source in a large, unstructured environment in one of the analog mission scenarios presented.

\section{ACKNOWLEDGMENT}

The authors wish to thank the Canadian Space Agency for their support, Western University and MDA Space Systems for their support, Clearpath Robotics for the Husky A200, and the Defence Research and Development Canada (DRDC) and the Natural Sciences and Engineering Research Council of Canada (NSERC) for their support.

\section{REFERENCES}

[1] M. Mumma, G. Villanueva, R. Novak, T. Hewagama, B. Bonev, M. DiSanti, A. Mandell, and M. Smith, "Strong release of methane on mars in northern summer 2003," Science, vol. 323, no. 5917, pp. 1041-1045, 2009.

[2] V. Formisano, S. Atreya, T. Encrenaz, N. Ignatiev, and M. Giuranna, "Detection of methane in the atmosphere of mars," Science, vol. 306, no. 5702, pp. 1758-1761, 2004.

[3] V. Krasnopolsky, J. Maillard, and T. Owen, "Detection of methane in the martian atmosphere: evidence for life?" Icarus, vol. 172, no. 2, pp. 537-547, 2004.

[4] T. Barfoot, G. D'Eleuterio, and A. Annan, "Subsurface surveying by a rover equipped with ground-penetrating radar," in Intell. Robots and Systems, 2003.(IROS 2003). Proc. 2003 IEEE/RSJ Int. Conf. on, vol. 3. IEEE, 2003, pp. 2541-2546.

[5] D. Wettergreen, N. Cabrol, V. Baskaran, F. Calderón, S. Heys, D. Jonak, R. Luders, D. Pane, T. Smith, J. Teza et al., "Second experiments in the robotic investigation of life in the atacama desert of chile," in Proc. 8th Int. Symp. on Artificial Intell., Robotics and Automation in Space, 2005.

[6] E. Tunstel, G. Anderson, and E. Wilson, "Motion trajectories for wide-area surveying with a rover-based distributed spectrometer," in Automation Congress, 2006. WAC'06. World. IEEE, 2006, pp. 1-8.

[7] G. Anderson, C. Sheesley, J. Tolson, E. Wilson, and E. Tunstel, "A mobile robot system for remote measurements of ammonia vapor in the atmosphere," in Systems, Man and Cybernetics, 2006. SMC'06. IEEE Int. Conf. on, vol. 1. IEEE, 2006, pp. 241-246.

[8] J. Tolson, C. Sheesley, S. Mohammed, S. Mahdi, I. Mohammad, E. Wilson, and E. Tunstel, "A system to sense nearsurface atmospheric gases of possible biological origin on mars," in Sensors, 2010 IEEE. IEEE, 2010, pp. 1858-1862.
[9] G. Anderson, E. Tunstel, and E. Wilson, "Robot system to search for signs of life on mars," Aerospace and Electronic Systems Magazine, IEEE, vol. 22, no. 12, pp. 23-30, 2007.

[10] E. Tunstel, G. Anderson, and E. Wilson, "Autonomous mobile surveying for science rovers using in situ distributed remote sensing," in Systems, Man and Cybernetics, 2007. ISIC. IEEE Int. Conf. on. IEEE, 2007, pp. 2348-2353.

[11] E. Tunstel, L. McFarlane, and V. Mejia, "Rover technology for open-path spectroscopy surveys," in Artificial Intell., Robotics, and Automation in Space. 9th Int. Symp. on, Los Angeles, CA, Feb. 2008, 2008.

[12] P. T. Furgale and T. D. Barfoot, "Visual teach and repeat for long-range rover autonomy," J. Field Robot., spec. issue on "Visual mapping and navigation outdoors", vol. 27, no. 5, pp. 534-560, 2010.

[13] B. Stenning and T. D. Barfoot, "Path planning on a network of paths," in Proc. of the IEEE Aerospace Conf., Big Sky, MT, Mar. 2011, pp. 1-12.

[14] S. M. LaValle and J. J. Kuffner, "Randomized kinodynamic planning," The Int. J. Robotics Research, vol. 20, no. 5, pp. 378-400, May 2001.

[15] B. Stenning, G. R. Osinski, T. D. Barfoot, G. Basic, M. Beauchamp, M. Daly, H. Dong, R. Francis, P. Furgale, J. Gammell, N. Ghafoor, A. Lambert, K. Leung, M. Mader, C. Marion, E. McCullough, C. McManus, J. Moores, and L. Preston, "Planetary surface exploration using a network of reusable paths," in Proc. of the 11th Int. Symp. on Artificial Intell., Robotics and Automation in Space (iSAIRAS), Turin, Italy, 4-6 September 2012.

[16] J. D. Gammell, C. Tong, and T. D. Barfoot, "Blinded by the light: Exploiting the deficiencies of a laser rangefinder for rover attitude estimation," in Proc. 10th Canadian Conf. on Comp. and Robot Vision (CRV), to appear., Regina, Saskatchewan, Canada, 28-31 May 2013.

[17] C. Tong, T. D. Barfoot, and E. Dupuis, "Three-dimensional SLAM for mapping planetary work site environments," $J$. Field Robot., Spec. issue on "Space Robotics", vol. 29, no. 3, pp. 381-412, May/June 2012.

[18] C. Chen, H. Hung, and J. Cheng, "RANSAC-based DARCES: A new approach to fast automatic registration of partially overlapping range images," IEEE Tran. Pattern Anal. Mach. Intell., vol. 21, no. 11, pp. 1229-1234, 1999.

[19] C. Tong and T. D. Barfoot, "Evaluation of heterogenous measurement outlier rejection schemes for robotic planetary worksite mapping," Acta Astronautica, 2012, available online on September 17, 2012.

[20] R. S. Merali, C. H. Tong, J. Gammell, J. Bakambu, E. Dupuis, and T. D. Barfoot, "Three-dimensional surface mapping using a semi-autonomous rover: A planetary analogue field experiment," in Proc. of the 11th Int. Symp. on Artificial Intell., Robotics and Automation in Space (iSAIRAS), Turin, Italy, 4-6 September 2012. 